\title{
A new boundary element algorithm for modeling and simulation of nonlinear thermal stresses in micropolar FGA composites with temperature-dependent properties
}

\author{
Mohamed Abdelsabour Fahmy ${ }^{1,2^{*}}$ (D)
}

\author{
*Correspondence: \\ maselim@uqu.edu.sa; \\ mohamed_fahmy@cisuez. \\ edu.eg \\ 1 Jamoum University College, \\ Umm Al-Qura University, \\ Alshohdaa 25371, Jamoum, \\ Mecca, Saudi Arabia \\ Full list of author information \\ is available at the end of the \\ article
}

\begin{abstract}
The main aim of this article is to develop a new boundary element method (BEM) algorithm to model and simulate the nonlinear thermal stresses problems in micropolar functionally graded anisotropic (FGA) composites with temperature-dependent properties. Some inside points are chosen to treat the nonlinear terms and domain integrals. An integral formulation which is based on the use of Kirchhoff transformation is firstly used to simplify the transient heat conduction governing equation. Then, the residual nonlinear terms are carried out within the current formulation. The domain integrals can be effectively treated by applying the Cartesian transformation method (CTM). In the proposed BEM technique, the nonlinear temperature is computed on the boundary and some inside domain integral. Then, nonlinear displacement can be calculated at each time step. With the calculated temperature and displacement distributions, we can obtain the values of nonlinear thermal stresses. The efficiency of our proposed methodology has been improved by using the communication-avoiding versions of the Arnoldi (CA-Arnoldi) preconditioner for solving the resulting linear systems arising from the BEM to reduce the iterations number and computation time. The numerical outcomes establish the influence of temperature-dependent properties on the nonlinear temperature distribution, and investigate the effect of the functionally graded parameter on the nonlinear displacements and thermal stresses, through the micropolar FGA composites with temperature-dependent properties. These numerical outcomes also confirm the validity, precision and effectiveness of the proposed modeling and simulation methodology.
\end{abstract}

Keywords: Boundary element method, Modeling algorithm, Nonlinear thermal stresses, Micropolar thermoelasticity, Functionally graded anisotropic composites, Cartesian transformation method

\section{Introduction}

Generalized thermoelasticity theories have attracted increased attention of many researchers in recent years due to their applications in many fields [1-4]. Functionally graded materials (FGMs) are a special kind of composite materials where their properties can be

- The Author(s) 2021. This article is licensed under a Creative Commons Attribution 4.0 International License, which permits use, sharing adaptation, distribution and reproduction in any medium or format, as long as you give appropriate credit to the original author(s) and the source, provide a link to the Creative Commons licence, and indicate if changes were made. The images or other third party material in this article are included in the article's Creative Commons licence, unless indicated otherwise in a credit line to the material. If material is not included in the article's Creative Commons licence and your intended use is not permitted by statutory regulation or exceeds the permitted use, you will need to obtain permission directly from the copyright holder. To view a copy of this licence, visit http://creativeco mmons.org/licenses/by/4.0/. 
tailored in accordance with the mechanical-technological properties required in different working conditions. FGMs are excellent advanced materials that change the manufacturing world for the better. FGMs have received a large amount of attention due to its ability to produce materials with tailored properties which are suitable candidates for several hightech applications such as graded structures on the atomic scale, graded hip implants, structural walls, sports equipment, design attractive interference colors for automobiles, etc. Then, the number of publications in this area of research has been growing exponentially in the past 20 years [5-10].

Temperature-dependent materials are those substances where the material properties depending upon the temperature at which they are measured, such as ice, bitumen, asphalt concrete, titanium, stainless steel, silver, gold, platinum, granular materials and reverse solubility polymers which are soluble in cold water and insoluble in hot water [11-17].

Recently, research on nonlinear thermal stresses problems has become very common because of its useful applications in several fields such as materials science, aircraft, astronautics, earthquake engineering, oceanology, geomechanics, aeronautics, plasma physics, nuclear reactors, Fiber-optic communication, etc. Because of mathematical challenges in solving these problems analytically, various numerical procedures have been developed to solve such problems. The boundary element method (BEM) [18-20] presents an interesting alternative to the more commonly used domain discretization methods such as finite difference method (FDM) [21-23], finite element method (FEM) [24-26] and finite volume method (FVM) [27-29]. The main advantage of BEM over domain methods is that only the boundary of the domain needs to be discretized, so, it has a major advantage over other methods that require the entire domain to be discretized [30-34]. This advantage has considerable importance for modeling nonlinear thermal stress problems utilizing BEM with very little computational cost and much less input data [35-43]. The BEM researchers developed the BEM procedures [44-60] to solve complex problems using several software, for example, FastBEM and ExaFMM.

In the present paper, a novel boundary element algorithm is proposed for modeling and simulation of nonlinear thermal stresses problems in micropolar FGA composites with temperature-dependent properties. In the proposed BEM formulation, the residual nonlinear terms are treated by using the Kirchhoff transformation and the domain integrals are treated by using the CTM. Then, at each time step the nonlinear temperature, displacements and thermal stresses are calculated at boundary nodes, and a few internal points which are considered to be utilized as initial values for another time step. The numerical findings investigate the effects of temperature-dependent properties and functionally graded parameter. These numerical findings also confirm the validity, precision and effectiveness of the proposed modeling and simulation methodology.

\section{Formulation of the problem}

Geometry of the current computational problem is shown in Fig. 1. The governing equations for nonlinear thermal stresses in micropolar composites with temperature-dependent properties can be expressed as

$$
\sigma_{i j, j}+\rho F_{i}=\rho \ddot{u}_{i}
$$




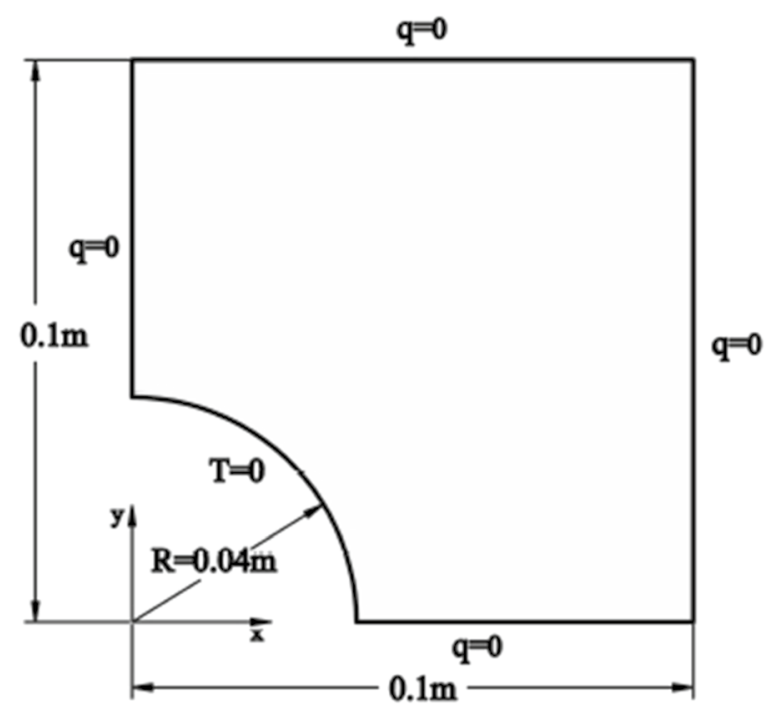

Fig. 1 Geometry of the considered problem

$$
\begin{aligned}
& m_{i j, j}+\varepsilon_{i j k} \sigma_{j k}+\rho M_{i}=J \rho \ddot{\omega}_{i} \\
& \nabla[\lambda(T) \nabla \mathrm{T}(X, t)]+h(X, T, t)=\rho(T) c(T) \frac{\partial}{\partial t} T(X, t)
\end{aligned}
$$

in which

$$
\begin{aligned}
& \sigma_{i j}=(x+1)^{m}\left[C_{i j k l} e \delta_{i j}+\breve{\alpha}\left(u_{j, i}-\varepsilon_{i j k} \omega_{k}\right)-\beta_{i j}\left(\theta+\tau_{1} \dot{\theta}\right)\right] \\
& m_{i j}=(x+1)^{m}\left[\alpha \omega_{k, k} \delta_{i j}+\bar{\alpha} \omega_{i, j}+\overline{\bar{\alpha}} \omega_{j, i}\right] \\
& \epsilon_{i j}=\varepsilon_{i j}-\varepsilon_{i j k}\left(r_{k}-\omega_{k}\right), \varepsilon_{i j}=\frac{1}{2}\left(u_{i, j}+u_{j, i}\right), \quad r_{i}=\frac{1}{2} \varepsilon_{i k l} u_{l, k}
\end{aligned}
$$

\section{BEM solution for temperature field}

The temperature governing equation can be reduced to a simpler form than that given in Eq. (3) by using Kirchhoff transformation which is defined as follows [14]

$$
\Theta=\int_{T_{0}}^{T} \frac{\lambda(\bar{T})}{\lambda_{0}} d \bar{T}
$$

By using Kirchhoff transformation, Eq. (3) can be written as follows

$$
\nabla^{2} \Theta(X, t)+\frac{1}{\lambda_{0}} h(X, \Theta, \mathrm{t})=\frac{\rho(\Theta) c(\Theta)}{\lambda(\Theta)} \frac{\partial \Theta(X, t)}{\partial t}
$$

where $\Theta$ and $\lambda_{0}$ are temperature function and thermal conductivity at $T_{0}$, respectively. 
Now, the right-hand side of (8) can be decomposed into linear and nonlinear parts as

$$
\nabla^{2} \Theta(X, t)+\frac{1}{\lambda_{0}} h(X, \Theta, \mathrm{t})=\frac{\rho_{0} c_{0}}{\lambda_{0}} \frac{\partial \Theta(X, t)}{\partial t}+N l(X, \Theta, \dot{\Theta})
$$

where $\lambda_{0}, \rho_{0}$ and $c_{0}$ are thermal conductivity, density and specific heat, respectively, at $T_{0}$.

where the nonlinear term can be written as

$$
N l(X, \Theta, \dot{\Theta})=\left[\frac{\rho(\Theta) c(\Theta)}{\lambda(\Theta)}-\frac{\rho_{0} c_{0}}{\lambda_{0}}\right] \dot{\Theta}
$$

According to [15, 16], Eq. (9) can be written as

$$
\nabla^{2} \Theta(X, t)+\frac{1}{\lambda_{0}} h_{N l}(X, \Theta, \dot{\Theta}, \mathrm{t})=\frac{\rho_{0} c_{0}}{\lambda_{0}} \frac{\partial \Theta(X, t)}{\partial t}
$$

where

$$
h_{N l}(X, \Theta, \dot{\Theta}, \mathrm{t})=h(X, \Theta, \mathrm{t})+\left[\rho_{0} c_{0}-\frac{\lambda_{0}}{\lambda(\Theta)} \rho(\Theta) c(\Theta)\right] \dot{\Theta}
$$

The integral equation which corresponds to Eq. (11) can be expressed as

$$
\begin{aligned}
C(P) \Theta\left(P, t_{n+1}\right)+a_{0} \int_{\Gamma} \int_{t_{n}}^{t_{n+1}} \Theta(Q, \tau) q^{*}\left(P, t_{n+1} ; Q, \tau\right) d \tau d \Gamma \\
=a_{0} \int_{\Gamma} \int_{t_{n}}^{t_{n+1}} q(Q, \tau) \Theta^{*}\left(P, t_{n+1} ; Q, \tau\right) d \tau d \Gamma \\
+\frac{a_{0}}{\lambda_{0}} \int_{\Omega} \int_{t_{n}}^{t_{n+1}} h_{N l}(Q, \Theta, \dot{\Theta}, \tau) \Theta^{*}\left(P, t_{n+1} ; Q, \tau\right) d \tau d \Omega \\
+\int_{\Omega} \Theta\left(Q, t_{n}\right) \Theta^{*}\left(P, t_{n+1} ; Q, t_{n}\right) d \Omega
\end{aligned}
$$

The fundamental solution and its normal derivative, respectively, can be written as

$$
\begin{aligned}
& \Theta^{*}\left(P, t_{n+1} ; Q, \tau\right)=\frac{1}{4 \pi a_{0}(t-\tau)} \exp \left[\frac{-r^{2}}{4 a_{0}(t-\tau)}\right] H(t-\tau) \\
& \mathrm{q}^{*}(P, t ; Q, \tau)=\frac{\partial}{\partial n} \Theta^{*}(P, t ; Q, \tau)=\frac{-r}{8 \pi a_{0}^{2}(t-\tau)^{2}} \exp \left[\frac{-r^{2}}{4 a_{0}(t-\tau)}\right] H(t-\tau) \frac{\partial r}{\partial n}
\end{aligned}
$$

where $a_{0}=\frac{\lambda_{0}}{\rho_{0} c_{0}}$ and $H$ is the Heaviside function.

The time integrals which corresponds to (14) and (15) can be computed analytically as

$$
\int_{t_{n}}^{t_{n+1}} \Theta^{*}\left(P, t_{n+1} ; Q, \tau\right) d \tau=\frac{1}{4 \pi a_{0}} E i\left(\frac{r^{2}}{4 a_{0} \Delta t}\right)
$$




$$
\int_{t_{n}}^{t_{n+1}} \mathrm{q}^{*}\left(P, t_{n+1} ; Q, \tau\right) d \tau=\frac{-1}{2 \pi a_{0} r} \exp \left(\frac{-r^{2}}{4 a_{0} \Delta t}\right) \frac{\partial r}{\partial n}
$$

where the exponential integral function $\operatorname{Ei}()$ can be defined as

$$
E i(\alpha)=\int_{\alpha}^{\infty} \frac{\exp (-x)}{x} d x
$$

The first domain integral of Eq. (13) contains the nonlinear term, that is

$$
I=\frac{a_{0}}{\lambda_{0}} \int_{\Omega}^{t_{n+1}} \int_{t_{n}}^{t_{N l}} h_{N l}(Q, \Theta, \dot{\Theta}, \tau) \Theta^{*}\left(P, t_{n+1} ; Q, \tau\right) d \tau d \Omega
$$

or

$$
I=\frac{a_{0}}{\lambda_{0}} \int_{\Omega} \int_{t_{n}}^{t_{n+1}}\left\{h(Q, \Theta, \tau)+\left[\rho_{0} c_{0}-\frac{\lambda_{0}}{\lambda(\Theta)} \rho(\Theta) c(\Theta)\right] \dot{\Theta}\right\} \Theta^{*}\left(P, t_{n+1} ; Q, \tau\right) d \tau \mathrm{d} \Omega
$$

By substituting the midpoint value of $h_{N l}$ and finite difference expression of $\dot{\Theta}$, we can write

$$
I_{N l}=\frac{1}{4 \pi \lambda_{0}} \int_{\Omega} h_{N I}\left(Q, \Theta_{n+0.5}, \dot{\Theta}_{n+0.5}, t_{n+0.5}\right) \operatorname{Ei}\left(\frac{r^{2}}{4 a_{0} \Delta t}\right) d \Omega
$$

where

$$
\begin{aligned}
& h_{N l}\left(Q, \Theta_{n+0.5}, t_{n+0.5}\right)=h\left(Q, \Theta_{n+0.5}, t_{n+0.5}\right)+\left[\rho_{0} c_{0}-\frac{\lambda_{0}}{\lambda\left(\Theta_{n+0.5}\right)} \rho\left(\Theta_{n+0.5}\right) c\left(\Theta_{n+0.5}\right)\right] \dot{\Theta}_{n+0.5} \\
& 2 C(P) \Theta\left(\mathrm{P}, t_{n+0.5}\right)-\frac{1}{2 \pi} \int_{\Gamma} \int_{t_{n}}^{t_{n+1}} \frac{\Theta\left(Q, t_{n+0.5}\right)}{r} \exp \left[\frac{-r^{2}}{4 a_{0} \Delta t}\right] \frac{\partial r}{\partial n} \mathrm{~d} \Gamma \\
& =\frac{1}{4 \pi} \int_{\Gamma} \mathrm{q}\left(Q, t_{n+0.5}\right) \operatorname{Ei}\left(\frac{r^{2}}{4 a_{0} \Delta t}\right) \mathrm{d} \Gamma \\
& \frac{1}{4 \pi a_{0}} \int_{\Omega} h_{N I}\left(Q, \Theta_{n+0.5}, \dot{\Theta}_{n+0.5}, t_{n+0.5}\right) \operatorname{Ei}\left(\frac{r^{2}}{4 a_{0} \Delta t}\right) \mathrm{d} \Omega \\
& \frac{1}{4 \pi a_{0} \Delta t} \int_{\Omega} \Theta\left(\mathrm{Q}, t_{n}\right) \exp \left(\frac{-r^{2}}{4 a_{0} \Delta t}\right) d \Omega+C(P) \Theta\left(\mathrm{P}, t_{n}\right)
\end{aligned}
$$

Now, we implement the CTM without domain discretisation to evaluate the domain integrals of (23). Thus, the unknown values at $M^{\prime}$ boundary nodes can be computed directly from the following system of matrix equations

$$
H \Theta^{\Gamma}=G Q^{\Gamma}+F+F_{N I}
$$


where $\Theta^{\Gamma}$ and $Q^{\Gamma}$ are $M^{\prime}$ dimension vectors contain boundary nodal values $\Theta$ and $q$, $F$ a vector depends on previous time step, $F_{N I}$ is a nonlinear term vector depends on unknown internal values, $H$ and $G$ are $M^{\prime} \times M^{\prime}$ dimension coefficient matrices. Also, the unknown values at $M^{\prime \prime}$ internal points may be calculated from the following system of matrix equations

$$
\Theta^{\Omega}=\hat{G} Q^{\Gamma}-\hat{H} \Theta^{\Gamma}+\hat{F}+\hat{F}_{N I}
$$

where $H, G, \hat{H}$ and $\hat{G}$ can be computed for all time steps. Also, $F, F_{N I}, \hat{F}$ and $\hat{F}_{N I}$ can be computed using CTM for all time steps.

The CTM method can be implemented to transform several domain integrals into boundary ones [47].

Now, we consider the following two-dimensional regular domain integral

$$
I=\int_{\Omega} p\left(x_{1}, x_{2}\right) \mathrm{d} \Omega
$$

By implementing Green's theorem as

$$
\int_{\Omega} \frac{\partial u\left(x_{1}, x_{2}\right)}{\partial x_{1}} \mathrm{~d} \Omega=\int_{\Gamma} u\left(x_{1}, x_{2}\right) \mathrm{d} x_{2}
$$

Now, we can write

$$
I=\int_{\Gamma} P_{1}\left(x_{1}, x_{2}\right) \mathrm{d} x_{2}
$$

where

$$
P_{1}\left(x_{1}, x_{2}\right)=\int_{\Gamma} p\left(x_{1}, x_{2}\right) \mathrm{d} x_{1}
$$

Since the integral in (29) cannot be determined analytically, so, we evaluate it numerically by the following integral equation

$$
P_{1}\left(x_{1}, x_{2}\right)=\int_{\alpha}^{x_{1}} p\left(x_{1}^{\prime}, x_{2}\right) \mathrm{d} x_{1}^{\prime}
$$

According to Khosravifard and Hematiyan [49], and using (30), the domain integral of (26) can be expressed as

$$
I=\int_{\Gamma}\left(\int_{\alpha}^{x_{1}} p\left(x_{1}^{\prime}, x_{2}\right) \mathrm{d} x_{1}^{\prime}\right) \mathrm{d} x_{2}
$$

where

$$
\alpha=\frac{x_{1 \min }+x_{1 \max }}{2}
$$


where $x_{1 \text { min }}$ and $x_{1 \text { max }}$ are minimum $x_{1}$ and maximum $x_{1}$ values, respectively.

The composite Gaussian quadrature method is applied to (26) yields

$$
I=\sum_{k=1}^{K} \int_{\Gamma_{k}} \int_{\alpha}^{x_{1}} \int_{\alpha}^{x_{1}} p\left(x_{1}^{\prime}, x_{2}\right) \mathrm{d} x^{\prime} \mathrm{d} x_{2}
$$

Equation (33) can be expressed as

$$
I=\sum_{k=1}^{K} J_{k} \sum_{i=1}^{N} w_{i} \sum_{l=1}^{L} J_{l} \sum_{j=1}^{J} w_{j} p\left(x_{1}\left(\eta_{j}\right), x_{2}\left(\eta_{i}\right)\right)
$$

where $J_{k}$ and $J_{l}$ are the transformation Jacobian for the $k$ th interval $l$ th interval, respectively, $K$ is the boundary elements number, $N$ and $J$ are the Gaussian integration points numbers of (33) for the outer integral and inner integral, respectively, $w_{i}$ and $w_{j}$ are Gauss points weights.

If $p$ described over a domain-boundary grid with irregularly spaced data. Then, by using the radial point interpolation method (RPIM) [50], the approximation using two-dimensional interpolation of the function $p$ may be written as

$$
p\left(x_{1}, x_{2}\right)=\sum_{i=1}^{M} \phi_{i}\left(x_{1}, x_{2}\right) p_{i}=\boldsymbol{\Phi}^{T} \mathrm{P}
$$

in which $\left(x_{1}, x_{2}\right)$ is any arbitrary point, $p_{i}$ is the $p$ value at $i$ and $\phi_{i}$ its shape function, $M$ (total number) $=M^{\prime}$ (boundary nodes number $)+M^{\prime \prime}$ (internal grid points number) . In the considered RPIM, the consistent shape functions are constructed using the radial basis functions. According to [50], the function $p\left(x_{1}, x_{2}\right)$ can be approximated as

$$
p(\mathbf{x})=\sum_{i=1}^{n} \alpha_{i} \psi_{i}(\mathbf{x})+\sum_{j=1}^{\bar{m}} b_{j} u_{j}(\mathbf{x})=\Psi^{T}(\mathbf{x}) \mathbf{a}+\mathbf{u}^{T}(\mathbf{x}) \mathbf{b}=\left[\Psi^{T}(\mathbf{x}) \mathbf{u}^{T}(\mathbf{x})\right]\left\{\begin{array}{l}
\mathbf{a} \\
\mathbf{b}
\end{array}\right\}
$$

The considered method is very simple for computation of regular and weakly singular domain integrals because all computations are performed in universal Cartesian coordinates, where kernels are defined by irregularly spaced data.

In order to create the RPIM shape functions, we apply the following Gaussian radial basis functions (GRBFs)

$$
\psi_{i}(\mathbf{x})=\exp \left[-a_{c}\left(\frac{R_{i}}{d_{c}}\right)^{2}\right]
$$

where $\psi_{i}$ are radial basis functions (RBFs), $n$ is the RBFs number, $\bar{m}$ is the polynomial basis functions number and $u_{j}(\mathbf{x})$, the augmented monomials and $\alpha_{i}$ and $b_{j}$ are unknown coefficients which can be evaluated from the following $n$ linear system of equations.

$$
\sum_{i=1}^{n} \alpha_{i} \psi_{i}\left(\mathbf{x}_{\mathbf{i}}\right)+\sum_{j=1}^{\bar{m}} b_{j} u_{j}\left(\mathbf{x}_{\mathbf{i}}\right)=p\left(\mathbf{x}_{\mathbf{i}}\right), i=1,2, \ldots, n
$$


and the following $\bar{m}$ linear constraints

$$
\sum_{i=1}^{n} \alpha_{i} u_{j}\left(\mathbf{x}_{\mathbf{i}}\right)=0, j=1,2, \ldots, \bar{m}
$$

From Eqs. (38) and (39), we can write $\alpha_{i}$ and $b_{j}$ in the following form

$$
\left\{\begin{array}{l}
\mathbf{a} \\
\mathbf{b}
\end{array}\right\}=\mathbf{B P}
$$

Based on [50], and using (40), Eq. (36) may be expressed as follows

$$
p(\mathbf{x})=\left[\boldsymbol{\psi}^{T}(\mathbf{x}) \mathbf{u}^{T}(\mathbf{x})\right] \mathbf{B P}=\phi^{T} \mathbf{P}
$$

where the matrix $\mathbf{P}$ is location- and geometry-dependent of boundary nodes and internal nodes.

where $\phi$ is the RPIM shape functions vector

$$
I=\sum_{k=1}^{K} J_{k} \sum_{i=1}^{N} w_{i} \sum_{l=1}^{L} J_{l} \sum_{j=1}^{J} w_{j} \sum_{r=1}^{M} p_{r} \phi_{r}\left(x_{1}\left(\eta_{j}\right), x_{2}\left(\eta_{i}\right)\right)
$$

Now, Eq. (42) can be expressed as

$$
I=\sum_{q=1}^{M} \gamma_{q} p_{q}=\boldsymbol{\gamma}^{\mathbf{T}} \mathbf{p}
$$

in which $\boldsymbol{\gamma}$ is the geometry- and location-dependent weight vector of grid points and $\mathbf{p}$ includes the values $p$ at boundary nodes and internal points.

\section{Regularization of BEM formulations and evaluation of the domain integrals}

In the BEM formulation of transient nonlinear thermal stresses problems, there are several regular and singular domain integrals with different kernels should be calculated with boundary-only discretization.

Now, we consider the following domain integrals which occur in the integral Eq. (23)

$$
\begin{aligned}
& I_{1}=\int_{\Omega} h_{N I}\left(Q, \Theta_{n+0.5}, \dot{\Theta}_{n+0.5}, t_{n+0.5}\right) E i\left(\frac{r^{2}}{4 a_{0} \Delta t}\right) \mathrm{d} \Omega \\
& I_{2}=\int_{\Omega} \Theta\left(Q, t_{n}\right) \exp \left[\frac{-r^{2}}{4 a_{0} \Delta t}\right] \mathrm{d} \Omega
\end{aligned}
$$

where the weakly singular exponential integral function $E i()$ in (44) can be written as

$$
E i(x)=\overline{E I}(x)-\ln (x)
$$

in which 


$$
\overline{E I}(x)=-0.57721566+\sum_{n=1}^{\infty}(-1)^{n-1} \frac{x^{n}}{n \cdot n !}
$$

Now, we can write Eq. (44) as

$$
\begin{aligned}
I_{1}= & \int_{\Omega} h_{N I}\left(Q, \Theta_{n+0.5}, \dot{\Theta}_{n+0.5}, t_{n+0.5}\right) \overline{E I}\left(\frac{r^{2}}{4 a_{0} \Delta t}\right) \mathrm{d} \Omega \\
& -\int_{\Omega} h_{N I}\left(Q, \Theta_{n+0.5}, \dot{\Theta}_{n+0.5}, t_{n+0.5}\right) \ln \left(\frac{r^{2}}{4 a_{0} \Delta t}\right) \mathrm{d} \Omega
\end{aligned}
$$

which can be written in the following form

$$
\begin{aligned}
I_{1}= & \int_{\Omega} h_{N I}\left(Q, \Theta_{n+0.5}, \dot{\Theta}_{n+0.5}, t_{n+0.5}\right)\left[\overline{E I}\left(\frac{r^{2}}{4 a_{0} \Delta t}\right)+\ln \left(4 a_{0} \Delta t\right)\right] \mathrm{d} \Omega \\
& +2 \int_{\Omega} h_{N I}\left(Q, \Theta_{n+0.5}, \dot{\Theta}_{n+0.5}, t_{n+0.5}\right) \ln \left(\frac{1}{r}\right) \mathrm{d} \Omega
\end{aligned}
$$

The weakly singular integral in Eq. (49) can be regularized as

$$
\begin{aligned}
I_{1}= & \int_{\Omega} h_{N I}\left(Q, \Theta_{n+0.5}, \dot{\Theta}_{n+0.5}, t_{n+0.5}\right)\left[\overline{E I}\left(\frac{r^{2}}{4 a_{0} \Delta t}\right)+\ln \left(4 a_{0} \Delta t\right)\right] \mathrm{d} \Omega \\
& +2 \int_{\Omega}\left[h_{N I}\left(Q, \Theta_{n+0.5}, \dot{\Theta}_{n+0.5}, t_{n+0.5}\right)-h_{N I}\left(P, \Theta_{n+0.5}, \dot{\Theta}_{n+0.5}, t_{n+0.5}\right)\right] \ln \left(\frac{1}{r}\right) \mathrm{d} \Omega \\
& +2 h_{N I}\left(P, \Theta_{n+0.5}, \dot{\Theta}_{n+0.5}, t_{n+0.5}\right) \int_{\Omega} \ln \left(\frac{1}{r}\right) \mathrm{d} \Omega
\end{aligned}
$$

Now, the first two integrals in (50) are regular, while the last domain integral can be transformed into a boundary. Therefore, Eq. (50) can be expressed as

$$
\mathrm{I}_{1}=\gamma^{\mathrm{T}}\left(\mathbf{p}_{1}+\mathbf{p}_{2}\right)+\mathrm{I}^{\prime}(\mathrm{P})
$$

where

$$
\mathrm{I}^{\prime}(\mathrm{P})=2 h_{N I}\left(P, \Theta_{n+0.5}, \dot{\Theta}_{n+0.5}, t_{n+0.5}\right) D_{1}(P)
$$

where

$$
D_{1}(P)=\int_{\Omega} \ln \left(\frac{1}{r}\right) d \Omega
$$

According to the application of CTM, the domain integral in (53) can be expressed as

$$
\left.D_{1}(P)=\int_{\Gamma}\left[\ln \left(\frac{1}{r}\right) \mathrm{d} x_{1}\right)\right] \mathrm{d} x_{2}=\int_{\Gamma}\left[-r_{1} \ln r-r_{2} \tan ^{-1}\left(\frac{r_{1}}{r_{2}}\right)+r_{1}\right] d x_{2}
$$


where $r_{i}=\left(x_{i}\right)_{Q}-\left(x_{i}\right)_{P}$ and $D_{1}(P)$ has the same value at each iteration of each time step.

The domain integral in (45) is regularized as

$$
I_{2}=\int_{\Omega}\left[\Theta\left(Q, t_{n}\right)-\Theta\left(P, t_{n}\right)\right] \exp \left[\frac{-r^{2}}{4 a_{0} \Delta t}\right] d \Omega+\Theta\left(P, t_{n}\right) \int_{\Omega} \exp \left[\frac{-r^{2}}{4 a_{0} \Delta t}\right] d \Omega
$$

The domain integrals in (55) can be evaluated using CTM, to allow us to write (55) as follows

$$
\mathrm{I}_{2}=\gamma^{\mathrm{T}} \mathbf{p}_{3}+\mathrm{I}^{\prime \prime}(\mathrm{P})
$$

where

$$
I^{\prime \prime}(P)=\Theta\left(P, t_{n}\right) \int_{\Gamma} \int \exp \left[\frac{-r^{2}}{4 a_{0} \Delta t}\right] d x_{1} d x_{2}
$$

which can be written as

$$
I^{\prime \prime}(P)=\Theta\left(P, t_{n}\right) D_{2}(P, \Delta t)
$$

where

$$
\begin{aligned}
D_{2}(P, \Delta t) & =\int_{\Gamma} \int_{\Gamma} \exp \left[\frac{-r^{2}}{4 a_{0} \Delta t}\right] d x_{1} d x_{2} \\
& =\sqrt{\pi a_{0} \Delta t} \int_{\Gamma} \exp \left(-\frac{r_{2}^{2}}{4 a_{0} \Delta t}\right) \operatorname{erf}\left(\frac{r_{1}}{2 \sqrt{a_{0} \Delta t}}\right) d x_{2}
\end{aligned}
$$

where the error function erf() can be expressed as

$$
\operatorname{erf}(a)=\frac{2}{\sqrt{\pi}} \int_{0}^{\alpha} \exp \left(-x^{2}\right) d x
$$

\section{BEM solution for displacement field}

The partial differential Eqs. (1) and (2), may be transformed into the following integral equations

$$
\begin{aligned}
& \int_{R}\left(\sigma_{i j, j}+U_{i}\right) u_{i}^{*} d R=0 \\
& \int_{R}\left(m_{i j, j}+\varepsilon_{i j k} \sigma_{j k}+V_{i}\right) \omega_{i}^{*} d R=0
\end{aligned}
$$

where

$$
U_{i}=\rho\left(F_{i}-\ddot{u}_{i}\right)
$$




$$
V_{i}=\rho\left(M_{i}-J \ddot{\omega}_{i}\right)
$$

in which $u_{i}^{*}$ and $\omega_{i}^{*}$ are weighting functions.

In the current paper, we considered the following boundary conditions

$$
\begin{aligned}
& u_{i}=\bar{u}_{i} \quad \text { on } S_{1} \\
& \lambda_{i}=\sigma_{i j} n_{j}=\bar{\lambda}_{i} \text { on } S_{2} \\
& \omega_{i}=\bar{\omega}_{i} \quad \text { on } S_{3} \\
& \mu_{i}=m_{i j} n_{j}=\bar{\mu}_{i} \text { on } S_{4}
\end{aligned}
$$

Applying integration by parts to Eqs. (61) and (62), we get

$$
\begin{aligned}
& -\int_{R} \sigma_{i j} u_{i, j}^{*} d R+\int_{R} U_{i} u_{i}^{*} d R=-\int \lambda_{i} u_{i}^{*} d S \\
& -\int_{R} m_{i j} \omega_{i, j}^{*} d R+\int_{R} \varepsilon_{i j k} \sigma_{j k} \omega_{i}^{*} d R+\int_{R} V_{i} \omega_{i}^{*} d R=-\int_{S_{4}} \mu_{i} \omega_{i}^{*} d S
\end{aligned}
$$

Based on Fahmy [41], we can write

$$
\begin{aligned}
& -\int_{R} \sigma_{i j, j} u_{i}^{*} d R+\int_{R}\left(m_{i j, j}+\varepsilon_{i j k} \sigma_{j k}\right) \omega_{i}^{*} d R+\int_{R} U_{i} u_{i}^{*} d R+\int_{R} V_{i} \omega_{i}^{*} d R \\
& =\int_{S_{2}}\left(\lambda_{i}-\bar{\lambda}_{i}\right) u_{i}^{*} d S+\int_{S_{1}}\left(\bar{u}_{i}-u_{i}\right) \lambda_{i}^{*} d S+\int_{S_{4}}\left(\mu_{i}-\bar{\mu}_{i}\right) \omega_{i}^{*} d S+\int_{S_{3}}\left(\bar{\omega}_{i}-\omega_{i}\right) \mu_{i}^{*} d S
\end{aligned}
$$

By using integration by parts for the left-hand side of (71), we obtain

$$
\begin{aligned}
& -\int_{R} \sigma_{i j} \varepsilon_{i j}^{*} d R-\int_{R} m_{i j, j} \omega_{i, j}^{*} d R+\int_{R} U_{i} u_{i}^{*} d R+\int_{R} V_{i} \omega_{i}^{*} d R \\
& =-\int_{S_{2}} \bar{\lambda}_{i} u_{i}^{*} d S-\int_{S_{1}} \lambda_{i} u_{i}^{*} d S+\int_{S_{1}}\left(\bar{u}_{i}-u_{i}\right) \lambda_{i}^{*} d S-\int_{S_{4}} \bar{\mu}_{i} \omega_{i}^{*} d S-\int_{S_{3}} \mu \omega_{i}^{*} d S+\int_{S_{3}}\left(\bar{\omega}_{i}-\omega_{i}\right) \mu_{i}^{*} d S
\end{aligned}
$$

On the basis of Fahmy [41], elastic and couple stresses can be written as

$$
\sigma_{i j}=\mathbb{A}_{i j k l} \varepsilon_{k l}, m_{i j}=\mathbb{B}_{i j k l} \omega_{k, l} \text { where } \mathbb{A}_{i j k l}=\mathbb{A}_{k l i j} \text { and } \mathbb{B}_{i j k l}=\mathbb{B}_{k l i j}
$$

Hence, Eq. (72) can be rewritten as

$$
\begin{aligned}
& -\int_{R} \sigma_{i j}^{*} \varepsilon_{i j} d R-\int_{R} m_{i j, j}^{*} \omega_{i, j} d R+\int_{R} U_{i} u_{i}^{*} d R+\int_{R} V_{i} \omega_{i}^{*} d R \\
& =-\int_{S_{2}} \bar{\lambda}_{i} u_{i}^{*} d S-\int_{S_{1}} \lambda_{i} u_{i}^{*} d S+\int_{S_{1}}\left(\bar{u}_{i}-u_{i}\right) \lambda_{i}^{*} d S-\int_{S_{4}} \bar{\mu}_{i} \omega_{i}^{*} d S-\int_{S_{3}} \mu_{i} \omega_{i}^{*} d S+\int_{S_{3}}\left(\bar{\omega}_{i}-\omega_{i}\right) \mu_{i}^{*} d S
\end{aligned}
$$


By applying integration by parts to the left-hand side of (74) for the second time, we get

$$
\begin{aligned}
& \int_{R} \sigma_{i j, j}^{*} u_{i} d R+\int_{R}\left(m_{i j, j}^{*}+\varepsilon_{i j k} \sigma_{j k}^{*}\right) \omega_{i} d R \\
& \quad=-\int_{S} u_{i}^{*} \lambda_{i} d S-\int_{S} \omega_{i}^{*} \mu_{i} d S+\int_{S} \lambda_{i}^{*} u_{i} d S+\int_{S} \mu_{i}^{*} \omega_{i} d S
\end{aligned}
$$

The weighting functions of $U_{i}=\Delta^{n}$ and $V_{i}=0$ along $e_{l}$ are obtained as follows:

$$
\begin{aligned}
& \sigma_{l j, j}^{*}+\Delta^{n} e_{l}=0 \\
& m_{i j, j}^{*}+\varepsilon_{i j k} \sigma_{j k}^{*}=0
\end{aligned}
$$

As Fahmy put it [41], the fundamental solution may be expressed as

$$
u_{i}^{*}=u_{l i}^{*} e_{l}, \omega_{i}^{*}=\omega_{l i}^{*} e_{l}, \lambda_{i}^{*}=\lambda_{l i}^{*} e_{l}, \mu_{i}^{*}=\mu_{l i}^{*} e_{l},
$$

Also, the weighting functions of $U_{i}=0$ and $V_{i}=\Delta^{n}$ along $e_{l}$ can be represented as:

$$
\begin{aligned}
& \sigma_{i j, j}^{* *}=0 \\
& m_{l j, j}^{* *}+\varepsilon_{l j k} \sigma_{j k}^{* *}+\Delta^{n} e_{l}=0
\end{aligned}
$$

Based on Fahmy [41], the fundamental solution can be expressed in the following form

$$
u_{i}^{*}=u_{l i}^{* *} e_{l}, \omega_{i}^{*}=\omega_{l i}^{* *} e_{l}, \lambda_{i}^{*}=\lambda_{l i}^{* *} e_{l}, \mu_{i}^{*}=\mu_{l i}^{* *} e_{l}
$$

Now, by considering the above two sets of weight functions into (75) we obtain

$$
\begin{aligned}
& C_{l i}^{n} u_{i}^{n}=-\int_{S} \lambda_{l i}^{*} u_{i} d S-\int_{S} \mu_{l i}^{*} \omega_{i} d S+\int_{S} u_{l i}^{*} \lambda_{i} d S+\int_{S} \omega_{l i}^{*} \mu_{i} d S \\
& C_{l i}^{n} \omega_{i}^{n}=-\int_{S} \lambda_{l i}^{* *} u_{i} d S-\int_{S} \mu_{l i}^{* *} \omega_{i} d S+\int_{S} u_{l i}^{* *} \lambda_{i} d S+\int_{S} \omega_{l i}^{* *} \mu_{i} d S
\end{aligned}
$$

Thus, we can write

$$
C^{n} \mathbb{q}^{n}=-\int_{S} \mathbb{p}^{*} \mathbb{q} d S+\int_{S} \mathbb{q}^{*} \mathbb{p} d S
$$

where 


$$
\begin{aligned}
& C^{n}=\left[\begin{array}{ll}
C_{11} & C_{12} \\
C_{21} & C_{22}
\end{array}\right], \mathbb{q}^{*}=\left[\begin{array}{lll}
u_{11}^{*} & u_{12}^{*} & \omega_{13}^{*} \\
u_{21}^{*} & u_{22}^{*} & \omega_{23}^{*} \\
u_{31}^{* *} & u_{32}^{* *} & \omega_{33}^{* *}
\end{array}\right], \mathbb{P}^{*}=\left[\begin{array}{lll}
\lambda_{11}^{*} & \lambda_{12}^{*} & \mu_{13}^{*} \\
\lambda_{21}^{*} & \lambda_{22}^{*} & \mu_{23}^{*} \\
\lambda_{31}^{* *} & \lambda_{32}^{* *} & \mu_{33}^{* *}
\end{array}\right], \mathbb{q}=\left[\begin{array}{l}
u_{1} \\
u_{2} \\
\omega_{3}
\end{array}\right], \mathbb{P} \\
&=\left[\begin{array}{l}
\lambda_{1} \\
\lambda_{2} \\
\mu_{3}
\end{array}\right]
\end{aligned}
$$

In order to solve (84) numerically, we use the following definitions

$$
\mathbb{q}=\psi \mathbb{q}^{j}, \mathbb{p}=\psi \mathbb{p}^{j}
$$

Substituting the above definitions and properties into (84) and discretizing the boundary into $N_{e}$ elements yield

$$
C^{n} \mathbb{q}^{n}=\sum_{j=1}^{N_{e}}\left[-\int_{\Gamma_{j}} \mathbb{P}^{*} \psi d \Gamma\right] \mathbb{q}^{j}+\sum_{j=1}^{N_{e}}\left[\int_{\Gamma_{j}} \mathbb{q}^{*} \psi d \Gamma\right] \mathbb{P}^{j}
$$

which can be expressed, after integration, as follows:

$$
C^{i} \mathbb{q}^{i}=-\sum_{j=1}^{N_{e}} \widehat{\mathbb{H}}^{i j} \mathbb{q}^{j}+\sum_{j=1}^{N_{e}} \widehat{\mathbb{G}}^{i j} \mathbb{P}^{j}
$$

Taking into consideration the following definition

$$
\mathbb{H}^{i j}=\left\{\begin{array}{l}
\hat{\mathbb{H}}^{i j} \quad \text { if } i \neq j \\
\hat{\mathbb{H}}^{i j}+C^{i} \quad \text { if } i=j
\end{array}\right.
$$

Therefore, depends upon the previous definition, Eq. (87) has the following form

$$
\sum_{j=1}^{N_{e}} \mathbb{H}^{i j} \mathbb{q}^{j}=\sum_{j=1}^{N_{e}} \widehat{\mathbb{G}}^{i j} \mathbb{P}^{j}
$$

which may be written as

$$
\mathbb{H} \mathbb{Q}=\mathbb{G P}
$$

Thus, we obtain

$$
\mathbb{A}=\mathbb{B}
$$

A time-stepping algorithm of Fahmy [54, 55] based on communication-avoiding Arnoldi preconditioner is implemented in order to obtain the temperature and displacement fields.

\section{Numerical results and discussion}

In the current paper, we considered the temperature-dependent pure copper material with the following physical data [61]: 
Table 1 Temperature dependent specific heat of pure copper [61]

\begin{tabular}{lllllll}
\hline $\mathrm{T}\left({ }^{\circ} \mathrm{C}\right)$ & 0 & 100 & 300 & 500 & 700 & 900 \\
$\mathrm{C}\left(\mathrm{J} / \mathrm{kg}{ }^{\circ} \mathrm{K}\right)$ & 385 & 397 & 417 & 433 & 451 & 480 \\
\hline
\end{tabular}

Table 2 Temperature dependent density of pure cupper [61]

\begin{tabular}{llrr}
\hline $\mathrm{T}\left({ }^{\circ} \mathrm{C}\right)$ & 0 & 500 & 900 \\
$\rho\left(\mathrm{kg} / \mathrm{m}^{3}\right)$ & 8930 & 8686 & 8458 \\
\hline
\end{tabular}

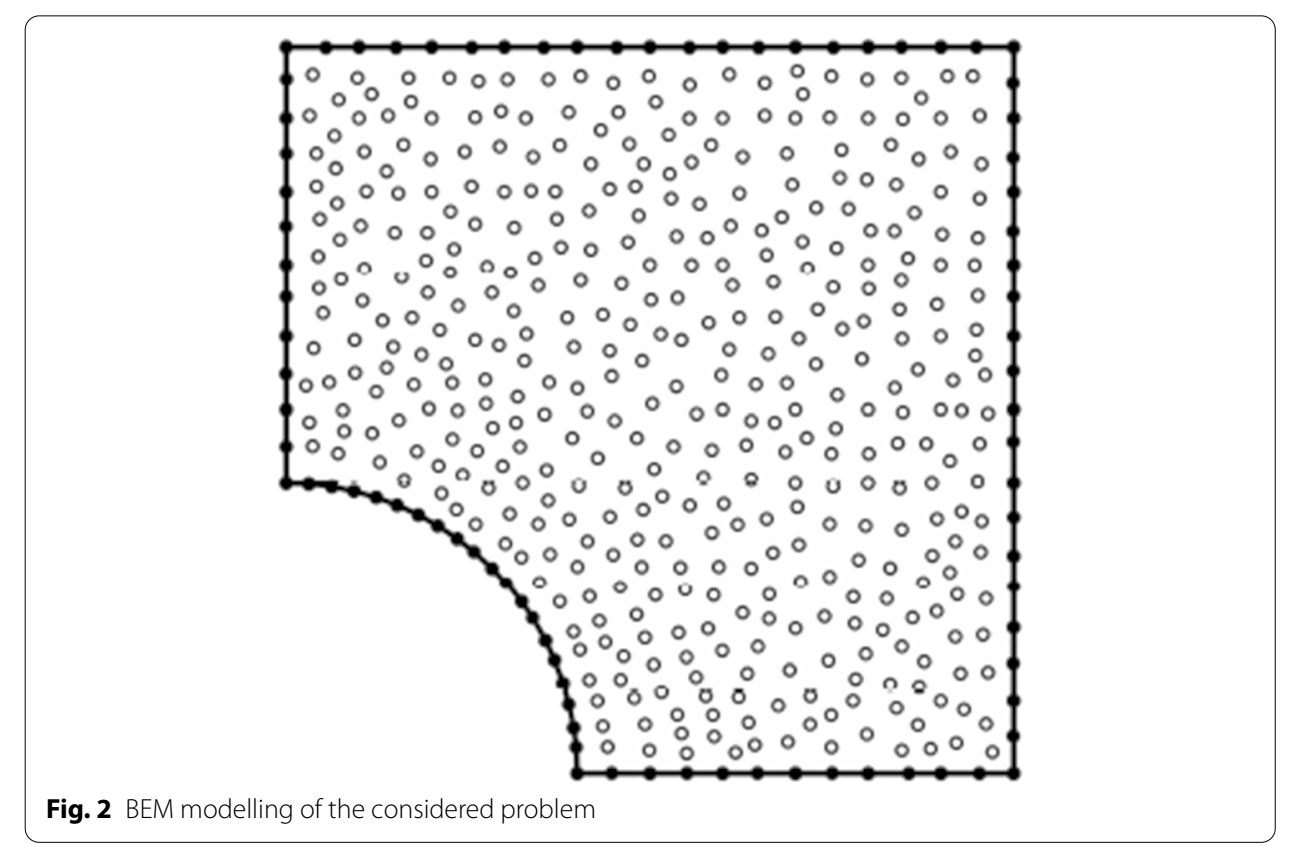

The thermal conductivity is

$$
\lambda=400\left(1-\frac{T}{6000}\right)
$$

The temperature dependent specific heat and density are shown in Tables 1 and 2, respectively.

The proposed BEM technique implemented in the current paper should be applicable to a wide range of nonlinear thermal stresses problems in micropolar FGA structures with temperature-dependent properties.

In the BEM modelling of the considered problem, the boundary has been discretized using 84 linear boundary elements and 404 internal points as shown in Fig. 2.

The effect of functionally graded parameter on the time distributions of the nonlinear displacements and thermal stresses plays an important role during the modeling process.

Figure 3 shows the time distribution of the nonlinear temperature for two different cases called temperature dependent (TD) and temperature independent (TID) 


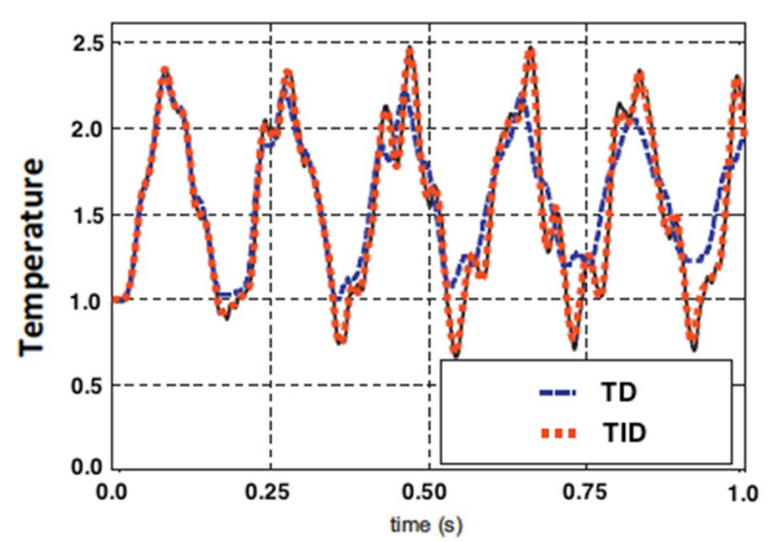

Fig. 3 Variation of the nonlinear temperature with time $\tau$

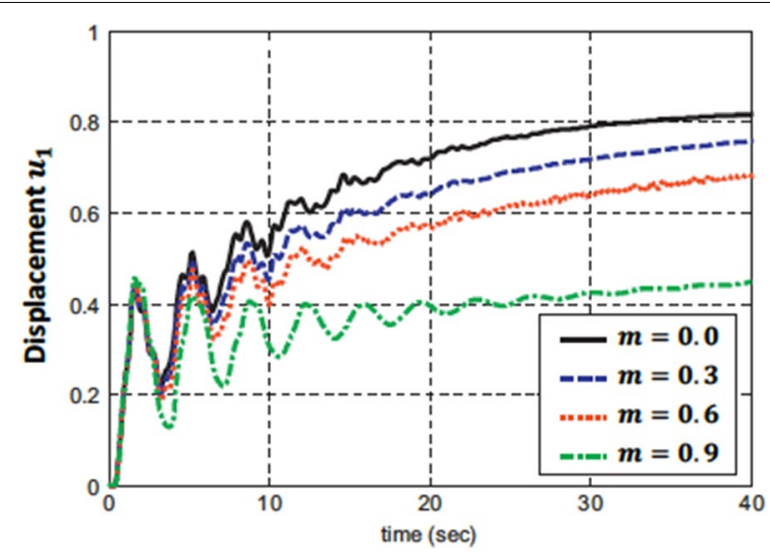

Fig. 4 Variation of the nonlinear displacement $u_{1}$ with time $\tau$ for different values of functionally graded parameter $m$

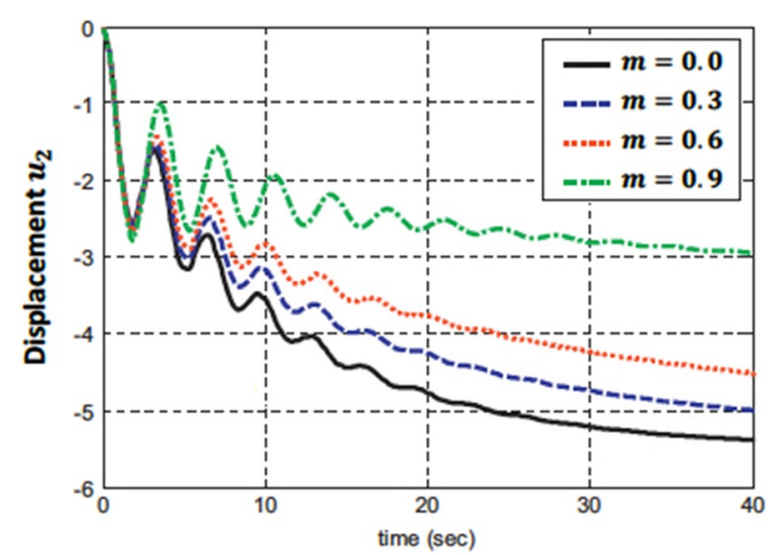

Fig. 5 Variation of the nonlinear displacement $u_{2}$ with time $\tau$ for different values of functionally graded parameter $m$

properties of micropolar FGA structures. It can be seen from this figure that it investigates the effect of temperature-dependent properties on the nonlinear temperature distribution. 


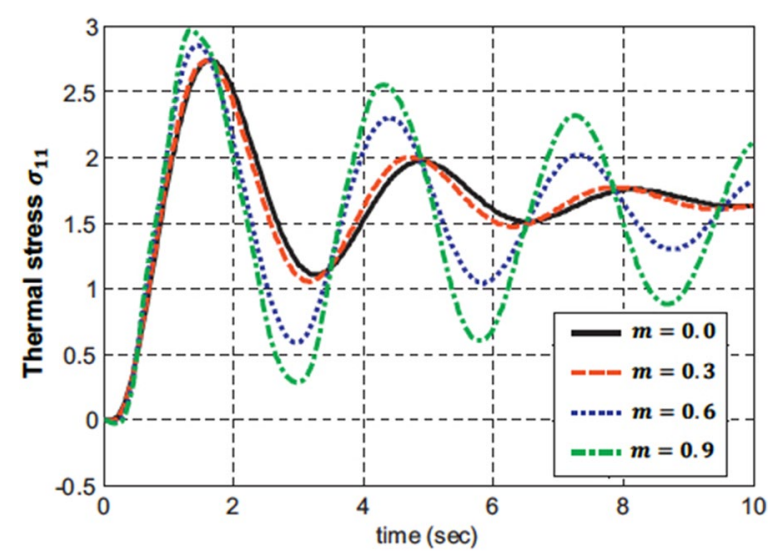

Fig. 6 Variation of the nonlinear thermal stress $\sigma_{11}$ with time $\tau$ for different values of functionally graded parameter $m$

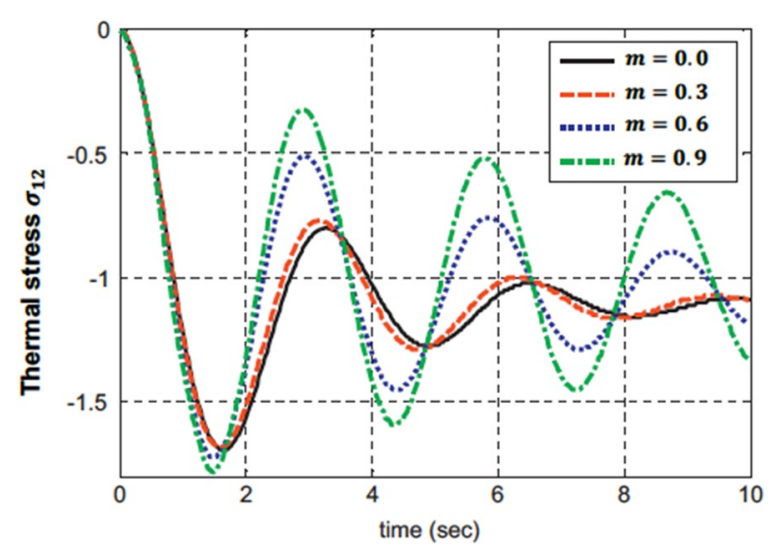

Fig. 7 Variation of the nonlinear thermal stress $\sigma_{12}$ with time $\tau$ for different values of functionally graded parameter $m$

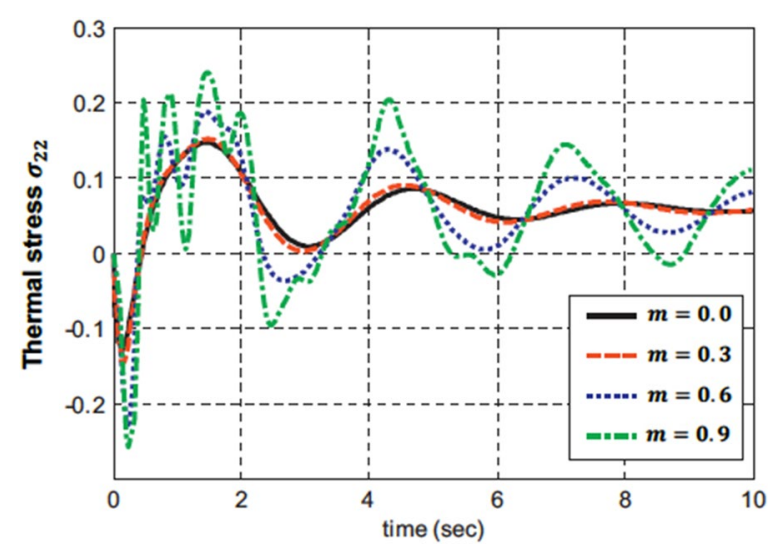

Fig. 8 Variation of the nonlinear thermal stress $\sigma_{22}$ with time $\tau$ for different values of functionally graded parameter $m$

Figures 4 and 5 show the time distributions of the nonlinear displacements $u_{1}$ and $u_{2}$ for homogeneous $(m=0)$ and functionally graded $(m=0.3,0.6$ and 0.9$)$ micropolar structures with temperature-dependent properties. It is clear from these figures that the 
functionally graded parameter has a tremendous impact on the displacements through the micropolar structures with temperature-dependent properties.

Figures 6, 7 and 8 show the time distributions of the nonlinear thermal stresses $\sigma_{11}$, $\sigma_{12}$ and $\sigma_{22}$ for homogeneous $(m=0)$ and functionally graded ( $m=0.3,0.6$ and 0.9$)$ micropolar structures with temperature-dependent properties. It can be shown from these figures that the functionally graded parameter has a considerable effect on the nonlinear thermal stresses through the micropolar structures with temperature-dependent properties.

The main characteristics of BEM over FDM or FEM, which make it the most appropriate numerical method, for dealing with the considered problem that has been performed are as follows [38, 41]:

- BEM does not need the internal domain to be discretized. But both FDM and FEM require the discretization of the whole domain. Therefore, BEM is more efficient and easy to use than FDM or FEM.

- BEM integration is a smoothing operation and is more numerically stable than differentiation operation of FDM or FEM. Therefore, all variables of the considered problem at any point are more precise than FDM or FEM.

- For the solution of the considered open boundary problem, BEM programmers deal with real geometry boundaries, while, FDM or FEM programmers deal with artificial boundaries, which are far away from the real problem. In addition, it is difficult for them to deal with the considered problem.

The efficiency of our proposed technique has been developed by using the communication-avoiding versions of the Arnoldi (CA-Arnoldi) preconditioner for solving the resulting linear systems arising from the BEM to reduce the iterations number and computation time.

The communication-avoiding versions of the Arnoldi (CA-Arnoldi) of Hoemmen [62] that also implemented by Fahmy [41], adaptive smoothing and prolongation algebraic multigrid (aSP-AMG) of Magri et al. [63] that also applied by Fahmy [43] and the regularized of Badahmane [64] which is also used by Fahmy [65] were compared with each other in Table 3. This table reports the iteration number (IT), CPU time, relative residual (RES) and error (ERR) of the tested iteration methods with respect to different values of

Table 3 Numerical results for the tested iteration methods

\begin{tabular}{llrlll}
\hline Time step & Method & IT & CPU & RES & ERR \\
\hline 0.005 & CA-Arnoldi & 30 & 0.0426 & $3.46 \mathrm{e}-07$ & $3.65 \mathrm{e}-09$ \\
& aSP-AMG & 60 & 0.0896 & $7.38 \mathrm{e}-07$ & $3.97 \mathrm{e}-07$ \\
& Regularized & 70 & 0.1032 & $8.79 \mathrm{e}-07$ & $4.76 \mathrm{e}-06$ \\
0.05 & CA-Arnoldi & 40 & 0.0876 & $1.38 \mathrm{e}-06$ & $4.18 \mathrm{e}-08$ \\
& aSP-AMG & 90 & 0.3241 & $2.95 \mathrm{e}-05$ & $6.73 \mathrm{e}-06$ \\
0.5 & Regularized & 110 & 0.4950 & $2.32 \mathrm{e}-05$ & $2.63 \mathrm{e}-05$ \\
& CA-Arnoldi & 50 & 0.2068 & $4.38 \mathrm{e}-05$ & $3.56 \mathrm{e}-07$ \\
& aSP-AMG & 260 & 0.8483 & $3.97 \mathrm{e}-04$ & $5.76 \mathrm{e}-05$ \\
& Regularized & 280 & 0.9235 & $2.94 \mathrm{e}-03$ & $6.49 \mathrm{e}-04$ \\
\hline
\end{tabular}




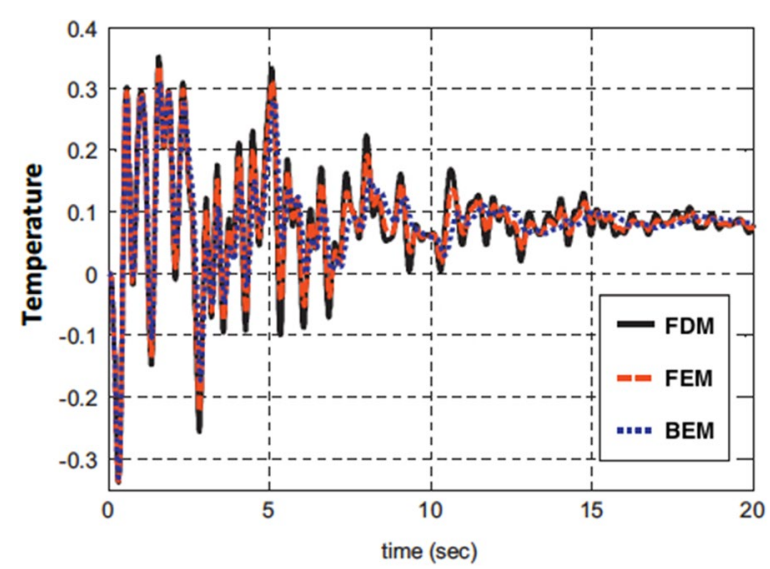

Fig. 9 Variation of the nonlinear temperature with time $\tau$ for FDM, FEM and BEM

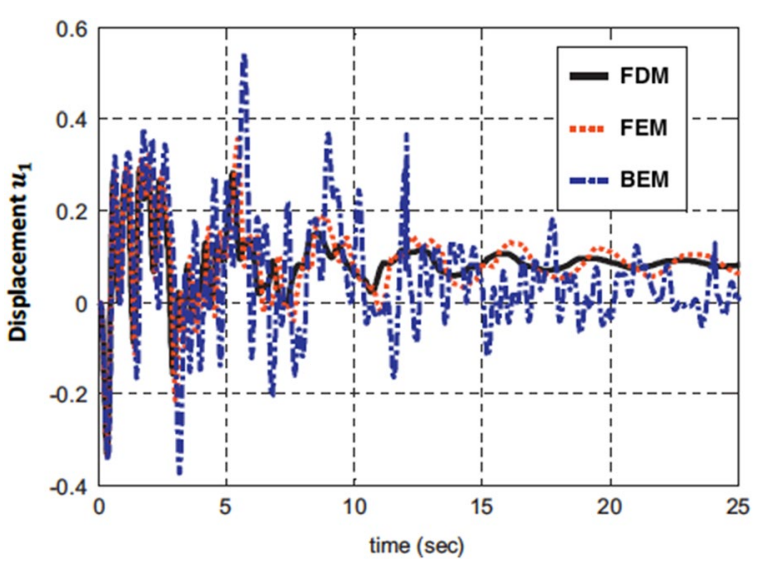

Fig. 10 Variation of the nonlinear displacement $u_{1}$ with time $\tau$ for FDM, FEM and BEM

time step. The results of this table show a superior efficiency of the CA-Arnoldi to other iterative methods.

\section{Numerical examples}

Due to the innovative character of the proposed technique, there are no available published results in the literature at present for comparison with the obtained BEM results. But, there are some literature can be considered to be special cases of our considered complex problem. Two numerical examples were studied to demonstrate the computational validity, accuracy and efficiency of the proposed BEM technique by comparing the numerical BEM outcomes with the FDM and FEM outcomes.

Example 1 Temperature and displacements of a square with a circular hole

In the considered special case, the boundary element model of the considered example, the boundary has been discretized using 84 linear boundary elements and 404 internal points as shown in Fig. 2, and the results of temperature and displacements are plotted in Figs. 9, 10, and 11. It can be seen from these figures that the BEM outcomes 


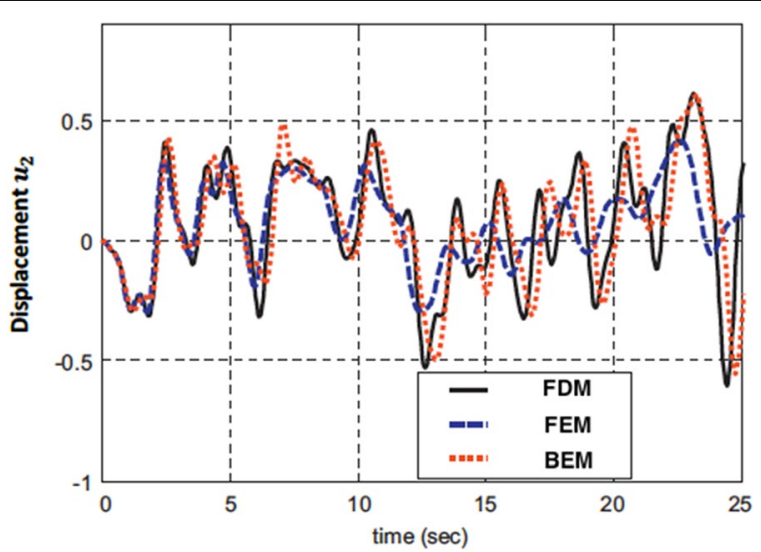

Fig. 11 Variation of the nonlinear displacement $u_{2}$ with time $\tau$ for FDM, FEM and BEM

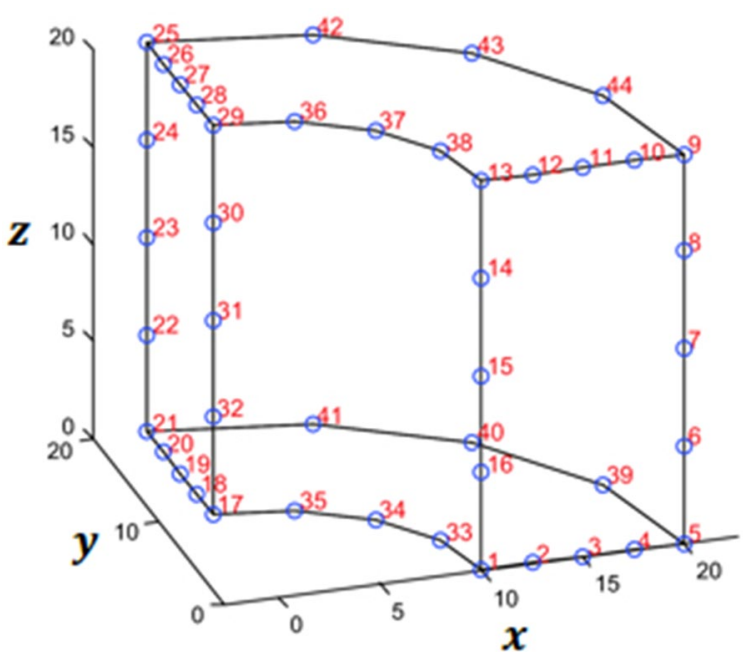

Fig. 12 Boundary element model of a thick hollow cylinder

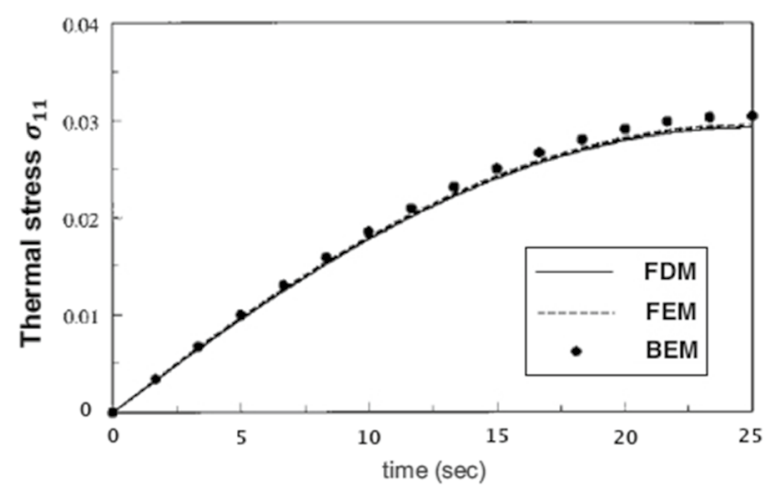

Fig. 13 Variation of the nonlinear thermal stress $\sigma_{11}$ with time $\tau$ for FDM, FEM and BEM 
are in very good agreement with the FDM outcomes of Awrejcewicz and Krysko [66] and FEM of Shakeriaski and Ghodrat [67].

Example 2 Nonlinear thermal stresses in a thick hollow cylinder

In the boundary element model of the considered example, the boundary has been discretized using 44 boundary elements and without internal points as shown in Fig. 12, and the results of nonlinear thermal stresses are plotted in Fig. 13. It can be seen from this figure that the BEM outcomes are in very good agreement with the FDM outcomes of Ahmad et al. [68] and FEM outcomes of Ibrahim and Gadisa [69].

\section{Conclusion}

A new boundary element modeling and simulation algorithm is developed based on time-dependent fundamental solutions in order to calculate the nonlinear thermal stresses in micropolar FGA composites, where material properties such as thermal conductivity, density and specific heat are assumed to be temperature-dependent. In the proposed BEM formulation, the boundary is subdivided into boundary elements, and the domain must be subdivided into internal cells, without any connectivity to increase the accuracy of the computation. There is no need to specify particular solutions for evaluating domain integrals, where the Galerkin meshfree method can be used to approximate the integral kernels of the domain integrals. The domain integrals are assessed effectively by the CTM integration weights which are chosen to be constant over each time step. The temperatures at the boundary nodes are the unknowns of the resulting equations system, whereas the internal points temperatures are calculated over all time steps without solving the whole equations system. The proposed iterative algorithm converges quickly and provides highly accurate numerical solution of the nonlinear without need of complex calculation. Comparing the results of the BEM with the FDM and FEM shows that BEM is effective and convenient. Also, it is predicated that BEM can be used in the analysis of strongly nonlinear thermoelastic problems accurately. During our treatment of the problem under consideration, we applied CA-Arnoldi, aSP-AMG and regularized preconditioners. The obtained numerical results demonstrate that CAArnoldi preconditioner has better performance than aSP-AMG and regularized preconditioners. The computational validity, accuracy and efficiency of the proposed technique were demonstrated. Hence, this paper demonstrates that, the proposed BEM technique based on CTM is faster and more accurate than FDM and FEM. Also, the proposed technique can be used in a wide variety of applications.

Nowadays, the knowledge of nonlinear thermal stresses in micropolar structures with temperature-dependent properties can be utilized by mechanical engineers in modeling of the solid composites, porous media and suspensions. As well as for chemists to detect the chemical reactions including adsorption and desorption.

Abbreviations

$\beta_{i j}$ : Stress-temperature coefficients; $\Gamma$ : Boundary of the considered region; $\boldsymbol{\gamma}$ : Weight vector; $\varepsilon_{i j}$ : Strain tensor; $\varepsilon_{i j k}$ : Permutation symbol: $\epsilon_{i j}$ : Micro-strain tensors; $\Theta$ : Function of temperature; $\Theta *$ : Fundamental solution; $\Theta^{\Gamma}: \Theta$ Values at boundary points; $\Theta^{\Omega}$ : $\Theta$ Values at internal points; $\lambda$ : Thermal conductivity; $\lambda_{0}$ : Thermal conductivity at $T_{0} ; \rho$ : Density; $\rho_{0}$ : Density at $T_{0} ; \sigma_{i j}$ : Force stress; $\phi_{i}\left(x_{1}, x_{2}\right)$ : Shape functions; $\phi$ : RPIM shape functions vector; $\psi_{i}(x)$ : Radial basis functions; $\omega_{i}$ : Micro-rotation; $\Omega$ : Domain of the considered region; $a_{c}$ : Support domain dimensionless size; $\boldsymbol{B}$ : Matrix 
depending on geometry and location; $c$ : Specific heat; $c_{0}$ : Specific heat at $\mathrm{T}_{0} ; C_{i j k l}$ : Constant elastic moduli; $C(P)$ : Local geometry coefficient; $d_{c}$ : Average spacing of grid points; $\operatorname{erf}()$ : Error function; $\boldsymbol{e}$ : Dilatation; $\operatorname{Ei}()$ : Exponential integral function; $F_{i}$ : Mass force; $h$ : Heat source function; $H$ and $G$ : Coefficient matrices; $J$ : Micro-inertia coefficient; $M_{i}$ : Mass couple; $\boldsymbol{m}_{i j}$ : Couple stress; $\boldsymbol{m}$ : Functionally graded parameter; $\bar{m}$ : Number of polynomial basis functions; $\boldsymbol{n}$ : Number of radial basis functions; $N l$ : Nonlinearity; $P$ : Collocation point; $Q$ : Field point; $q$ : Normal derivative of $\Theta ; q^{*}$ : Normal derivative of $\Theta^{*} ; R_{i}$ : Distance from field point to grid point; $r$ : Euclidian distance between $\mathrm{P}$ and $\mathrm{Q} ; t$ : Time; $T$ : Temperature; $T_{0}$ : Reference temperature; $\boldsymbol{u}_{i}$ : Displacement; $\boldsymbol{x}$ : Field point; $\boldsymbol{x}_{i}$ : Grid point.

\section{Acknowledgements}

None.

\section{Authors' contributions}

The author read and approved the final manuscript.

\section{Funding}

None.

Availability of data and materials

All numerical setting data are presented in the article.

\section{Declartions}

\section{Competing interests}

The author declares no competing interests.

\section{Author details}

${ }^{1}$ Jamoum University College, Umm Al-Qura University, Alshohdaa 25371, Jamoum, Mecca, Saudi Arabia. ${ }^{2}$ Faculty of Computers and Informatics, Suez Canal University, New Campus, 4.5 Km, Ring Road, El Salam District, Ismailia 41522, Egypt.

Received: 3 August 2020 Accepted: 9 March 2021

Published online: 26 March 2021

\section{References}

1. Sherief HH, Ezzat MA. Solution of the generalized problem of thermoelasticity in the form of series of functions. J Therm Stress. 1994;17:75-95.

2. Ezzat MA. Fundamental solution in generalized magneto-thermoelasticity with two relaxation times for perfect conductor cylindrical region. Int J Eng Sci. 2004;13-14:1503-19.

3. Ezzat MA, Awad ES. Constitutive relations, uniqueness of solution, and thermal shock application in the linear theory of micropolar generalized thermoelasticity involving two temperatures. J Therm Stress. 2010;33:226-50.

4. Ezzat MA, El-Bary AA. Memory-dependent derivatives theory of thermo-viscoelasticity involving two-temperature. J Mech Sci Technol. 2015;10:4273-9.

5. Fahmy MA. Implicit-explicit time integration DRBEM for generalized magneto-thermoelasticity problems of rotating anisotropic viscoelastic functionally graded solids. Eng Anal Bound Elem. 2013;37:107-15.

6. Fahmy MA. A computerized DRBEM model for generalized magneto-thermo-visco-elastic stress waves in functionally graded anisotropic thin film/substrate structures. Latin Am J Solids Struct. 2014;1 1:386-409.

7. Fahmy MA. A new LRBFCM-GBEM modeling algorithm for general solution of time fractional order dual phase lag bioheat transfer problems in functionally graded tissues. Numer Heat Transfer A Appl. 2019:75:616-26.

8. Fahmy MA. Generalized magneto-thermo-viscoelastic problems of rotating functionally graded anisotropic plates by the dual reciprocity boundary element method. J Therm Stress. 2013;36:1-20.

9. Fahmy MA. A three-dimensional generalized magneto-thermo-viscoelastic problem of a rotating functionally graded anisotropic solids with and without energy dissipation. Numer Heat Transfer A Appl. 2013;63:713-33.

10. Fahmy MA. Shape design sensitivity and optimization of anisotropic functionally graded smart structures using bicubic B-splines DRBEM. Eng Anal Bound Elem. 2018;87:27-35.

11. Tauchert TR. Thermal stresses in an orthotropic cylinder with temperature dependent elastic properties. Dev Theor Appl Mech. 1976;8:201-12.

12. Sugano $Y$. Analysis of transient thermal stresses in an orthotropic finite rectangular plate exhibiting temperaturedependent material properties by finite difference method. Trans Jpn Soc Mech Eng Ser A. 1983;49:1315-23.

13. Ezzat M, Zakaria M, Abdel-Bary A. Generalized thermoelasticity with temperature dependent modulus of elasticity under three theories. J Appl Math Comput. 2004;14:193-212.

14. Othman MIA, Lotfy K, Farouk RM. Generalized thermo-microstretch elastic medium with temperature dependent properties for different theories. Eng Anal Bound Elem. 2010;34:229-37.

15. Wang $Y$, Zhang $X$, Liu D. Asymptotic analysis of generalized thermoelasticity for axisymmetric plane strain problem with temperature-dependent material properties. Int J Appl Mech. 2013;5:1350023.

16. Fahmy MA, Shaw S, Mondal S, Abouelregal AE, Lotfy K, Kudinov IA, Soliman AH. Boundary element modeling for simulation and optimization of three-temperature anisotropic micropolar magneto-thermoviscoelastic problems in porous smart structures using NURBS and genetic algorithm. Int J Thermophys. 2021;42:29.

17. Demirbas MD, Ekici R, Apalak MK. Thermoelastic analysis of temperature-dependent functionally graded rectangular plates using finite element and finite difference methods. Mech Adv Mater Struct. 2020;27:707-24. 
18. Matsumoto T, Guzik A, Tanaka M. A boundary element method for analysis of thermoelastic deformations in materials with temperature dependent properties. Int J Numer Meth Eng. 2005;64:1432-58.

19. Goto T, Suzuki M. A boundary integral equation method for nonlinear heat conduction problems with temperaturedependent material properties. Int J Heat Mass Transf. 1996;39:823-30.

20. Amado JM, Tobar MJ, Ramil A, Yáñez A. Application of the Laplace transform dual reciprocity boundary element method in the modelling of laser heat treatments. Eng Anal Bound Elem. 2005;29:126-35.

21. Peng HS, Chen CL. Hybrid differential transformation and finite difference method to annular fin with temperaturedependent thermal conductivity. Int J Heat Mass Transf. 2011;54:2427-33.

22. Demirbas MD. Thermal stress analysis of functionally graded plates with temperature-dependent material properties using theory of elasticity. Compos B Eng. 2017;131:100-24.

23. Carollo LFS, Silva ALFL, Silva SMML. A dierent approach to estimate temperature-dependent thermal properties of metallic materials. Materials. 2019;12:2579.

24. Rüberg T, Cirak F, Aznar JMG. An unstructured immersed finite element method for nonlinear solid mechanics. Adv Model Simul Eng Sci. 2016;3:22.

25. Habib F, Sorelli L, Fafard M. Full thermo-mechanical coupling using eXtended finite element method in quasi-transient crack propagation. Adv Model Simul Eng Sci. 2018;5:18.

26. Sowmya G, Gireesha BJ, Madhu M. Analysis of a fully wetted moving fin with temperature-dependent internal heat generation using the finite element method. Heat Transf. 2020;49:1939-54.

27. Sobamowo G, Ogunmola BY, Nzebuka GC. Finite volume method for analysis of convective longitudinal fin with temperature-dependent thermal conductivity and internal heat generation. Defect Diffus Forum. 2017;374:106-20

28. Gong J, Xuan L, Ying B, Wang $\mathrm{H}$. Thermoelastic analysis of functionally graded porous materials with temperaturedependent properties by a staggered finite volume method. Compos Struct. 2019;224:111071.

29. Dilip DG, John G, Panda S, Mathew J. Finite-volume-based conservative numerical scheme in cylindrical coordinate system to predict material removal during micro-EDM on Inconel 718. J Braz Soc Mech Sci Eng. 2020;42:90.

30. El-Naggar AM, Abd-Alla AM, Fahmy MA, Ahmed SM. Thermal stresses in a rotating non-homogeneous orthotropic hollow cylinder. Heat Mass Transf. 2002;39:41-6.

31. Abd-Alla AM, El-Naggar AM, Fahmy MA. Magneto-thermoelastic problem in non-homogeneous isotropic cylinder. Heat Mass Transf. 2003;39:625-9.

32. El-Naggar AM, Abd-Alla AM, Fahmy MA. The propagation of thermal stresses in an infinite elastic slab. Appl Math Comput. 2003;12:220-6.

33. Eskandari AH, Baghani M, Sohrabpour S. A time-dependent finite element formulation for thick shape memory polymer beams considering shear effects. Int J Appl Mech. 2019;10:1850043.

34. Soliman AH, Fahmy MA. Range of applying the boundary condition at fluid/porous interface and evaluation of beavers and Joseph's slip coefficient using finite element method. Computation. 2020;8:14.

35. Fahmy MA. A new BEM for fractional nonlinear generalized porothermoelastic wave propagation problems. In: Computers materials and continua. Encino: Tech Science Press; 2021.

36. Fahmy MA. Shape design sensitivity and optimization for two-temperature generalized magneto-thermoelastic problems using time-domain DRBEM. J Therm Stress. 2018:41:119-38.

37. Fahmy MA. Boundary element algorithm for modeling and simulation of dual-phase lag bioheat transfer and biomechanics of anisotropic soft tissues. Int J Appl Mech. 2018;10:1850108.

38. Fahmy MA. Modeling and optimization of anisotropic viscoelastic porous structures using CQBEM and moving asymptotes algorithm. Arab J Sci Eng. 2019;44:1671-84.

39. Fahmy MA. Boundary element modeling and simulation of biothermomechanical behavior in anisotropic laserinduced tissue hyperthermia. Eng Anal Bound Elem. 2019;101:156-64.

40. Fahmy MA. Design optimization for a simulation of rotating anisotropic viscoelastic porous structures using timedomain OQBEM. Math Comput Simul. 2019;66:193-205.

41. Fahmy MA. A new boundary element strategy for modeling and simulation of three temperatures nonlinear generalized micropolar-magneto-thermoelastic wave propagation problems in FGA structures. Eng Anal Bound Elem. 2019;108:192-200.

42. Fahmy MA. A new convolution variational boundary element technique for design sensitivity analysis and topology optimization of anisotropic thermo-poroelastic structures. Arab J Basic Appl Sci. 2020;27:1-12.

43. Fahmy MA. Boundary element algorithm for nonlinear modeling and simulation of three temperature anisotropic generalized micropolar piezothermoelasticity with memory-dependent derivative. Int J Appl Mech. 2020;12:2050027.

44. Brebbia CA, Telles JCF, Wrobel L. Boundary element techniques in engineering. New York: Springer; 1984.

45. Wrobel LC, Brebbia CA. The dual reciprocity boundary element formulation for nonlinear diffusion problems. Comput Methods Appl Mech Eng. 1987:65:147-64.

46. Partridge PW, Brebbia CA. Computer implementation of the BEM dual reciprocity method for the solution of general field equations. Commun Appl Numer Methods. 1990;6:83-92.

47. Hematiyan MR. Exact transformation of a wide variety of domain integrals into boundary integrals in boundary element method. Commun Numer Methods Eng. 2008;24:1497-521.

48. Mohammadi M, Hematiyan MR, Marin L. Boundary element analysis of nonlinear transient heat conduction problems involving non-homogenous and nonlinear heat sources using time-dependent fundamental solutions. Eng Anal Bound Elem. 2010;34:655-65.

49. Khosravifard A, Hematiyan MR. A new method for meshless integration in $2 D$ and $3 D$ Galerkin meshfree methods. Eng Anal Bound Elem. 2010;34:30-40.

50. Liu GR, Gu YT. An introduction to meshfree methods and their programming. New York: Springer; 2005.

51. Fahmy MA. Thermoelastic stresses in a rotating non-homogeneous anisotropic body. Numer Heat Transf A Appl. 2008;53:1001-11.

52. Abd-Alla AM, Fahmy MA, El-Shahat TM. Magneto-thermo-elastic problem of a rotating non-homogeneous anisotropic solid cylinder. Arch Appl Mech. 2008;78:135-48. 
53. Fahmy MA, El-Shahat TM. The effect of initial stress and inhomogeneity on the thermoelastic stresses in a rotating anisotropic solid. Arch Appl Mech. 2008;78:431-42.

54. Fahmy MA. A time-stepping DRBEM for magneto-thermo-viscoelastic interactions in a rotating nonhomogeneous anisotropic solid. Int J Appl Mech. 2011;3:1-24.

55. Fahmy MA. A time-stepping DRBEM for the transient magneto-thermo-visco-elastic stresses in a rotating nonhomogeneous anisotropic solid. Eng Anal Bound Elem. 2012;36:335-45.

56. Fahmy MA. Numerical modeling of transient magneto-thermo-viscoelastic waves in a rotating nonhomogeneous anisotropic solid under initial stress. Int J Model Simul Sci Comput. 2012;3:1250002.

57. Fahmy MA. Transient magneto-thermo-viscoelastic stresses in a rotating nonhomogeneous anisotropic solid with and without a moving heat source. J Eng Phys Thermophys. 2012;85:950-8.

58. Fahmy MA. Transient magneto-thermo-elastic stresses in an anisotropic viscoelastic solid with and without moving heat source. Numer Heat Transf A Appl. 2012;61:547-64.

59. Fahmy MA. Transient magneto-thermoviscoelastic plane waves in a non-homogeneous anisotropic thick strip subjected to a moving heat source. Appl Math Model. 2012;36:4565-78.

60. Fahmy MA. The effect of rotation and inhomogeneity on the transient magneto-thermoviscoelastic stresses in an anisotropic solid. ASME J Appl Mech. 2012;79:1015.

61. Green D, Perry R. Perry's chemical engineer's handbook. 8th ed. New York: Mc Graw Hill; 2007.

62. Hoemmen M. Communication-avoiding Krylov subspace methods. Ph.D. Dissertation. University of California, Berkeley; 2010.

63. Magri VP, Franceschini A, Janna C. A novel algebraic multi-grid approach based on adaptive smoothing and prolongation for ill-conditioned systems. SIAM J Sci Comput. 2019;41:A190-219.

64. Badahmane A. Regularized preconditioned GMRES and the regularized iteration method. Appl Numer Math. 2020;152:159-68.

65. Fahmy MA. A novel BEM for modeling and simulation of 3T nonlinear generalized anisotropic micropolar-thermoelasticity theory with memory dependent derivative. CMES. 2021;126:175-99.

66. Awrejcewicz J, Krysko VA. Elastic and thermoelastic problems in nonlinear dynamics of structural members: applications of the bubnov-galerkin and finite difference methods. New York: Springer International Publishing; 2020.

67. Shakeriaski F, Ghodrat M. The nonlinear response of Cattaneo-type thermal loading of a laser pulse on a medium using the generalized thermoelastic model. Theor Appl Mech Lett. 2020;10:1-12.

68. Ahmad F, Almatroud AO, Hussain S, Farooq SE, Ullah R. Numerical solution of nonlinear Diff. equations for heat transfer in micropolar fluids over a stretching domain. Mathematics. 2020;8:854.

69. Ibrahim W, Gadisa G. Finite element analysis of couple stress micropolar nanofluid flow by non-Fourier's law heat flux model past stretching surface. Heat Transf Asian Res. 2019;48:3763-89.

\section{Publisher's Note}

Springer Nature remains neutral with regard to jurisdictional claims in published maps and institutional affiliations.

\section{Submit your manuscript to a SpringerOpen ${ }^{\circ}$ journal and benefit from:}

- Convenient online submission

- Rigorous peer review

- Open access: articles freely available online

- High visibility within the field

- Retaining the copyright to your article

Submit your next manuscript at $\boldsymbol{\nabla}$ springeropen.com 